\title{
Ganho de peso e alterações metabólicas em camundongos submetidos à dieta hiperlipídica
}

\author{
Weight gain and metabolic changes in mice submitted to high fat diet
}

Juliana Lopes Pereiraa, Priscilla Cristovam Sampaio e Souzaa, Vivian Izumi Shinzatoa, Sandramara Sasso ${ }^{b}$, Bruna Larissa Spontoni do Espirito Santo ${ }^{b}$, Lidiani Figueiredo Santana ${ }^{b}$, Tamy Ingrid Restelc, Karine de Cássia Freitas ${ }^{\mathrm{d}}$

a Acadêmica do Curso de Nutrição da Universidade Federal de Mato Grosso do Sul, Campo Grande, MS, Brasil.

b Nutricionista. Mestre em Saúde e Desenvolvimento na Região Centro-Oeste. Universidade Federal de Mato Grosso do Sul, Campo Grande, MS, Brasil.

Bióloga. Mestre em Saúde e Desenvolvimento na Região Centro-Oeste. Técnica do Biotério Central. Instituto de Biociências. Universidade Federal de

Mato Grosso do Sul, Campo Grande, MS, Brasil.

d Nutricionista. Doutora em Ciências. Docente na Faculdade de Ciências Farmacêuticas, Alimentos e Nutrição. Universidade Federal de Mato Grosso do Sul,

Campo Grande, MS, Brasil.

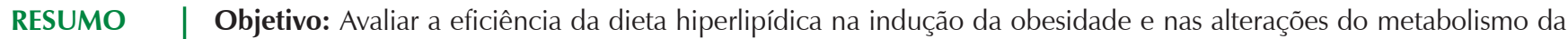
glicose e dos lipídeos, em camundongos C57BL/6.

Materiais e Métodos: Utilizou-se 19 camundongos C57BL/6, divididos em grupo controle (dieta comercial; $\mathrm{n}=9$ ) e experimental (dieta hiperlipídica; $n=10$ ). Foi mensurado o consumo de ração e ganho de peso durante 12 semanas, e calculado o Coeficiente de Eficiência Alimentar (CEA) e Índice Aterogênico. Foi realizado o Teste Oral de Tolerância à Glicose (TOTG), e construída a curva glicêmica, sendo verificada a área sobre a curva (AUC). Foram dissecados o fígado e os sítios de tecido adiposo (TA), feita análise histológica do fígado e TA epididimal, calculado o Índice de Adiposidade (IA\%) e mensurada a concentração do colesterol total e frações, glicose e triglicerídeos séricos.

Resultados: O grupo experimental apresentou menor consumo de ração $(p<0,001)$ e maior ganho de peso total $(p=0,001)$ e CEA $(p<0,001)$. Observou-se aumento no TA epididimal $(p<0,001)$, retroperitoneal $(p<0,001)$, mesentérico $(p=0,002)$ e perirrenal $(p=0,012)$, gordura total $(p<0,001)$ e IA $\%(p<0,001)$ no grupo experimental. O colesterol total foi maior no grupo experimental $(p=0,025)$ e o HDL menor no controle $(p=0,005)$. A área dos adipócitos do grupo experimental foi maior que do controle $(p=0,049)$. Nos demais parâmetros não houve diferença significante entre os grupos.

Conclusão: Este modelo induziu a obesidade em um curto período de tempo e promoveu aumento da adiposidade visceral, com dieta de baixo custo. São necessários outros estudos visando melhorar sua eficiência e alterar de forma completa os lipídeos sanguíneos e a glicemia nos camundongos C57BL/6.

Palavras-chave: modelos animais; obesidade; transtornos do metabolismo da glicose; transtornos do metabolismo dos lipídeos.

Objective: The aim of this study was to evaluate the efficiency of high fat diet in the induction of obesity and glucose and lipid metabolism disorders in C57BL/6 mice.

Materials and Methods: Animals were organized into two groups, totalizing 19 C57BL/6 mice: control group (commercial diet; $n=9$ ) and experimental group (high fat diet; $n=10$ ). Food intake and weight gain were measured during 12 weeks and Food Efficiency Coefficient (FEC) and Aterogenic Index were calculated. The Oral Glucose Tolerance Test (TOTG) was performed, the glycemic curve was constructed and the area under the curve (AUC) was verified. Liver and adipose tissue (AT) sites were dissected and histological analysis of the liver and epididymal adipose tissue were performed. The Adiposity Index (Al\%) was calculated and the concentration of total cholesterol, its fractions, glucose and serum triglycerides were measured.

Results: The experimental group presented lower food intake $(p<0.001)$ and higher total weight gain $(p=0.001)$ and FEC $(p<0.001)$. It was observed in the experimental group an increase in the epididymal $(p<0.001)$, retroperitoneal $(p<0.001)$, mesenteric $(p=0.002)$, perirenal AT $(p=0.012)$, total fat $(p<0.001)$ and Al\% $(p<0.001)$. Total cholesterol was higher in the experimental group $(p=0.025)$ and the HDL lower in the control group $(p=0.005)$. The adipocyte area of the experimental group was also higher than the control group $(p=0.049)$. In the other parameters there was no significant difference between groups.

Conclusion: This model induced obesity in a short period of time and promoted increased visceral adiposity with a low-cost diet. Further studies are needed to improve its efficiency and to completely change blood lipids and glycemia in C57BL/6 mice.

Keywords: models animal; obesity; glucose metabolism disorders; lipid metabolism disorders. 


\section{INTRODUÇÃO}

A obesidade consiste em uma doença crônica, ocasionada por diversos fatores etiológicos que favorecem o aumento excessivo de tecido adiposo no organismo ${ }^{1}$. O acúmulo de gordura, principalmente na região abdominal, é determinante para o risco de desenvolvimento de diversas patologias, como hipertensão arterial, dislipidemias, resistência à insulina, diabetes mellitus e síndrome metabólica² ${ }^{2}$ O estilo de vida atual, onde há facilidade de acesso e baixo custo de alimentos palatáveis e com grande densidade calórica e um baixo nível de atividade física diária, tem sido apontado como o principal responsável pelo aumento da prevalência dessa patologia ${ }^{3}$. Dados da vigilância de fatores de risco e proteção para doenças crônicas não transmissíveis obtidos por inquérito telefônico (VIGITEL), demonstraram que entre as capitais brasileiras o percentual de adultos obesos aumentou de 11,8\% em 2006 para 18,9\% em 2015.

Para melhor entender a fisiopatologia da obesidade e suas complicações metabólicas tornou-se necessário o uso de modelos experimentais que visam determinar de maneira controlada a contribuição e a relação de cada um dos componentes dessa disfunção e fornecer subsídios para sua prevenção e/ou tratamento ${ }^{5}$. Os diferentes modelos experimentais que buscam induzir em animais a obesidade de forma semelhante à humana podem ser classificados, em sua maioria, em modelos genéticos e induzidos por dieta $^{5}$. Contudo, são raras as causas genéticas como fatores etiológicos da obesidade humana ${ }^{6}$.

O modelo que mais se assemelha à patogênese da obesidade humana é o induzido por dieta, onde se oferece ao animal um aporte calórico maior, através da sobrecarga de carboidratos ou lipídeos. Na maioria dos estudos é utilizada a ração padrão $\mathrm{AIN}-93^{7}$, de alto custo, à qual se acrescenta alimentos hipercalóricos, como o leite condensado, sacarose, chocolate, amendoim e biscoito ${ }^{8}$, o que encarece ainda mais os custos com o experimento. Porém, na prática experimental, ainda assim, muitos desses modelos são limitados, visto que pode ocorrer baixa aceitação da dieta pelos animais, ausência de obesidade abdominal e alterações no metabolismo da glicose e dos lipídeos sanguíneos ${ }^{9}$, com grande variação no tempo necessário para indução das alterações desejadas ${ }^{5,10}$. Ao mesmo tempo, ainda são amplamente discutidas na literatura as respostas metabólicas ocasionadas pelo consumo de dieta hiperlipídica ${ }^{11}$.

Assim, este estudo teve por objetivo avaliar a eficiência de uma dieta hiperlipídica de baixo custo na indução da obesidade e dos transtornos metabólicos supracitados, em camundongos da linhagem isogênica C57BL/6.

\section{MATERIAIS E MÉTODOS}

Foram utilizados 19 camundongos da linhagem isogênica C57BL/6, machos, saudáveis, com 8-10 semanas de vida, obtidos do Biotério Central da UFMS. Os animais permaneceram em adaptação ao ambiente de ensaio por cinco dias e após esse período foram divididos em dois grupos: controle com 9 animais, que receberam dieta comercial (Ração Nuvilab $^{\circledR}$ ), e experimental com 10 animais, que receberam dieta hiperlipídica (Ração Nuvilab ${ }^{\circledR}+$ banha de porco $)^{10}$, e água ad libitum durante 12 semanas, e foram mantidos em caixas para criação de roedores contendo 2-3 animais, sob temperatura de $22 \pm 2^{\circ} \mathrm{C}$, com ciclo claro-escuro de $12 \mathrm{~h}$.

A dieta hiperlipídica foi elaborada através da mistura da Ração Nuvilab ${ }^{\circledR}$ triturada (62\%) à banha de porco, da marca Sadia $^{\circledR}$, adquirida no comércio local (38\%). Com base no rótulo dos ingredientes, a mesma constituiu-se de $60,3 \%$ de lipídeos, 30,60\% de carboidratos e 9,10\% de proteínas, enquanto a ração comercial apresentou 8,57\% de lipídeos, $70,48 \%$ de carboidratos e 20,95\% de proteínas.

A cada dois dias eram colocados 100 gramas de ração da dieta comercial ou hiperlipídica em cada caixa e o consumo individual diário dos animais era obtido pela média do consumo (descontado sobra da ração) durante os dois dias, dividido pelo número de animais por caixa. O peso dos animais foi aferido semanalmente. A partir desses dados foi calculado o ganho de peso semanal e o Coeficiente de Eficiência Alimentar (CEA), por meio da equação: CEA= ganho de peso (g) semanal/consumo alimentar (g) semanal ${ }^{12}$.

Ao final das 12 semanas, foi realizado o Teste Oral de Tolerância à Glicose (TOTG) $)^{13}$. Oito horas antes do teste, os animais foram colocados em jejum. No início do teste foi retirada uma amostra de sangue a partir de uma incisão na cauda dos animais e o valor obtido foi o T0 (tempo zero). Subsequentemente, uma solução de D-glicose (2g/ $\mathrm{kg}$ de peso) foi administrada oralmente por gavagem. Posteriormente, a leitura da glicemia foi realizada nos tempos 15, 30, 60 e 120 min após a aplicação da glicose. As dosagens da glicemia foram realizadas utilizando o glicosímetro Sens II $\left(\right.$ Injex $\left.{ }^{\circledR}\right)$, de acordo com as especificações do fabricante. A partir do TOTG foi construída a curva glicêmica e verificada a área sobre a curva (AUC), no software GraphPad Prism.

Três dias após o TOTG e jejum de 12 horas, os animais foram submetidos à eutanásia. Os animais foram anestesiados com xilazina e quetamina (10 mg/kg:75 mg/kg) e o sangue retro-orbital foi retirado através de um capilar e recolhido em um tubo com gel separador de soro. As amostras de sangue foram centrifugadas a $3000 \mathrm{rpm}$ por 5 minutos e o soro armazenado para posterior análises. Os animais foram 
colocados em câmara de $\mathrm{CO}_{2}$ para confirmação da eutanásia. Quando não havia mais a presença de reflexos, os animais foram abertos para a retirada dos cinco sítios de tecido adiposo (epididimal, omental, mesentérico, retroperitoneal e perirrenal), que foram pesados, assim como o fígado. Foi calculado o Índice de Adiposidade (\%), utilizando a seguinte fórmula: gordura total/peso corporal final ${ }^{14}$.

Para análise histológica foi retirada uma amostra do fígado e do tecido adiposo epididimal, as quais foram guardadas em cassetes e colocadas em uma solução de formol a 10\%. Após a fixação em líquido de Bouin, os espécimes foram desidratados em baterias de álcool etílico, diafanizados em xilol, impregnados e incluídos em parafina, cortados em micrótomo com espessura de $5 \mu \mathrm{m}$ e corados com Hematoxilina-Eosina ${ }^{15}$. As imagens das lâminas foram capturadas utilizando o sistema de câmera digital Leica DFC 495, integrado ao microscópio Leica DM5500 B, em aumento de 20x. Os campos histológicos de fígado foram analisados quanto à presença de esteatose hepática por um sistema teste composto por 36 pontos, no programa STEPanizer, o qual é utilizado para estimar a densidade de volume (Vv) de esteatose hepática não alcoólica, a partir da contagem de pontos que tocavam as gotículas de gordura nos hepatócitos: $V_{v}=P p / P t$, onde Pp é o número de pontos que se sobrepõem a esteatose e Pt é o número de pontos totais do sistema (36 pontos) ${ }^{16,17}$. Para área dos adipócitos, foram selecionados campos representativos no corte histológico, onde se mediu a área de 100 adipócitos por amostra utilizando-se o software Image Pro Plus, e o resultado final representa a média da área desses 100 adipócitos.

Foi mensurada a concentração do colesterol (total e frações), glicose e triglicerídeos (TG) séricos, de acordo com o kit comercial LabTest Diagnóstica ${ }^{\circledR}$, Brasil. Para o cálculo das concentrações bioquímicas $(\mathrm{mg} / \mathrm{dL})$ foram aplicadas as seguintes fórmulas: absorbância do teste/absorbância do padrãox 200, para obter os valores de triglicerídeos e colesterol total; absorbância do teste/absorbância do padrãox 100, para glicose; absorbância do teste/absorbância do padrãox 40, para HDL; triglicerídeos/5, para VLDL; e Colesterol Total - (HDL+VLDL), para LDL. Para calcular o Índice Aterogênico foi utilizada a seguinte fórmula: colesterol total/ $\mathrm{HDL}^{18}$.

Esta pesquisa foi submetida ao Comitê de Ética no Uso de Animais (CEUA) da Universidade Federal de Mato Grosso do Sul (UFMS) e aprovada sob o Protocolo no 636/2014, estando de acordo com os princípios éticos adotados pelo Conselho Nacional de Controle de Experimentação Animal (CONCEA), com a legislação vigente e demais disposições da ética em investigação que envolve diretamente os animais.
Os resultados foram expressos em média \pm erro padrão da média (EPM) e analisados usando o software GraphPad Prism e SigmaStat, aplicando-se o test $t$ e análise de variância (ANOVA) de duas vias, com pós-teste de Bonferroni. Valores de $p \leq 0,05$ foram considerados estatisticamente significantes.

\section{RESULTADOS}

O grupo experimental apresentou consumo de ração menor $(p<0,001)$ e maior ganho de peso total $(p=0,001)$ e CEA $(p<0,001)$ que o grupo controle (Tabela 1). Não houve diferença estatística no peso inicial entre os grupos $(p=0,954)$, sendo que a partir da quinta semana já se observou aumento significante no peso dos animais do grupo experimental $(p=0,020)$, que se manteve até o final do experimento $(p<0,001)$ (Figura 1).

Tabela 1. Consumo de ração, peso inicial e final, ganho de peso e CEA de camundongos alimentados com dieta comercial e dieta hiperlipídica.

\begin{tabular}{lccc}
\hline & \multicolumn{2}{c}{ Grupos de estudo } & \\
\cline { 2 - 3 } Parâmetros & $\begin{array}{c}\text { Dieta } \\
\text { Comercial } \\
(\mathbf{n}=\mathbf{9})\end{array}$ & $\begin{array}{c}\text { Dieta } \\
\text { Hiperlipídica } \\
(\mathbf{n}=\mathbf{1 0})\end{array}$ & $\boldsymbol{p}$ \\
Peso inicial (g) & $24,67 \pm 0,47$ & $24,60 \pm 0,99$ & 0,954 \\
Peso final (g) & $29,44 \pm 0,65$ & $38,20 \pm 1,74$ & $<0,001$ \\
Ganho de peso semanal (g) & $0,40 \pm 0,03$ & $0,99 \pm 0,16$ & 0,003 \\
Ganho de peso total (g) & $4,78 \pm 0,32$ & $12,22 \pm 1,04$ & 0,001 \\
Consumo total de ração (g) & $269,19 \pm 5,94$ & $220,51 \pm 7,51$ & $<0,001$ \\
CEA & $0,02 \pm 0,00$ & $0,07 \pm 0,01$ & $<0,001$ \\
\hline
\end{tabular}

CEA: Coeficiente de Eficiência Alimentar.

Valores representados em média \pm erro padrão da média. Teste $t$ de Student.

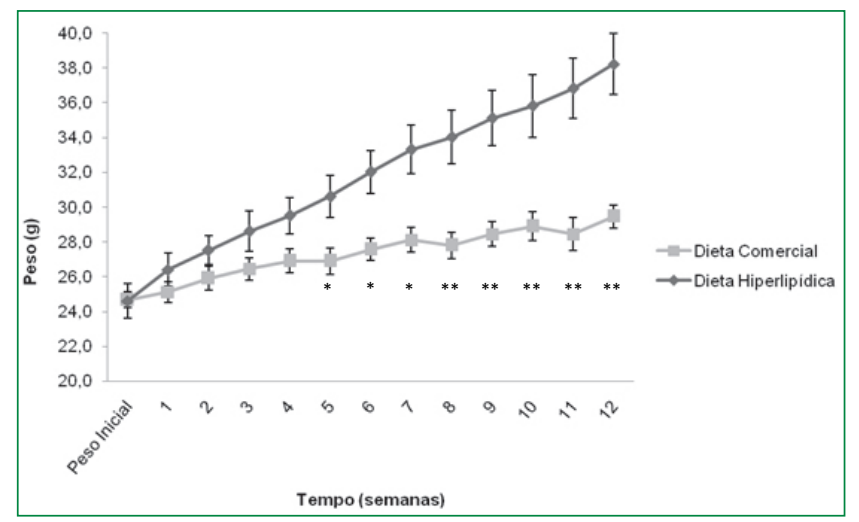

Figura 1. Ganho de peso semanal (média \pm erro padrão da média) dos grupos alimentados com dieta comercial e dieta hiperlipídica.

${ }^{*} p<0,05 ;{ }^{*} p<0,001$. ANOVA de duas vias, pós-teste de Bonferroni.

Foi observado que o grupo experimental apresentou maiores sítios de tecido adiposo epididimal $(p<0,001)$, retroperitoneal $(p<0,001)$, mesentérico $(p=0,002)$ e 
perirrenal $(p=0,012)$, sendo o somatório de gordura dos cinco sítios (incluindo omental) $(p<0,001)$ e o Índice de Adiposidade $(p<0,001)$ superior nesse grupo em comparação ao grupo controle, e o peso do fígado não diferiu entre os grupos $(p=0,553)$, conforme Tabela 2.

O colesterol total foi maior no grupo experimental $(p=0,025)$ e o HDL menor no grupo controle $(p=0,005)$. Foram maiores no grupo experimental os níveis de glicemia (21,02\%), triglicerídeos (14,46\%), VLDL (14,93\%) e LDL $(45,15 \%)$, porém sem diferença significante, $p=0,200$, $p=0,177, p=0,167$ e $p=0,157$, respectivamente (Tabela 3). O Índice Aterogênico não apresentou diferença significante $(p=0,406)$, sendo 3,5 no grupo controle e 3,1 no grupo experimental.

$\mathrm{Na}$ análise histológica observou-se que a área dos adipócitos do grupo experimental era maior $(p=0,049)$ do que a do grupo controle, respectivamente, 5.601,0 $\mu \mathrm{m}^{2} \mathrm{e}$ $2.005,7 \mu \mathrm{m}^{2}$. Na análise histológica do fígado os dois grupos apresentaram esteatose hepática, sem diferença entre eles

Tabela 2. Peso do fígado e dos cinco sítios de tecido adiposo (TA) de camundongos alimentados com dieta comercial e dieta hiperlipídica.

\begin{tabular}{lccc}
\hline & \multicolumn{2}{c}{ Grupos de estudo } & \\
Parâmetros & $\begin{array}{c}\text { Dieta } \\
\text { Comercial } \\
(\mathbf{n = 9 )}\end{array}$ & $\begin{array}{c}\text { Dieta } \\
\text { Hiperlipídica } \\
(\mathbf{n = 1 0 )}\end{array}$ & $\boldsymbol{p}$ \\
\hline Fígado (g) & $\mathbf{1 , 1 3 \pm 0 , 0 3}$ & $\mathbf{1 , 0 1 \pm 0 , 1 8}$ & 0,553 \\
TA Epididimal (g) & $0,33 \pm 0,02$ & $1,62 \pm 0,24$ & $<0,001$ \\
TA Omental (g) & $0,06 \pm 0,02$ & $0,07 \pm 0,01$ & 0,865 \\
TA Retroperitoneal (g) & $0,10 \pm 0,01$ & $0,66 \pm 0,08$ & $<0,001$ \\
TA Mesentérico (g) & $0,21 \pm 0,02$ & $0,91 \pm 0,18$ & 0,002 \\
TA Perirrenal (g) & $0,05 \pm 0,01$ & $0,23 \pm 0,06$ & 0,012 \\
Gordura total (g) & $0,76 \pm 0,05$ & $3,50 \pm 0,37$ & $<0,001$ \\
Índice de adiposidade (\%) & $2,57 \pm 0,15$ & $8,95 \pm 0,60$ & $<0,001$ \\
\hline
\end{tabular}

Valores representados em média \pm erro padrão da média.

Teste $t$ de Student.

Tabela 3. Glicemia de jejum e lipídeos séricos de camundongos alimentados com dieta comercial e dieta hiperlipídica.

\begin{tabular}{lccc}
\hline & \multicolumn{2}{c}{ Grupos de estudo } & \\
\cline { 2 - 4 } Parâmetros & $\begin{array}{c}\text { Dieta } \\
\text { Comercial } \\
(\mathbf{n = 8})\end{array}$ & $\begin{array}{c}\text { Dieta } \\
\text { Hiperlipídica } \\
(\mathbf{n = 8})\end{array}$ & $\boldsymbol{p}$ \\
Glicemia de jejum (mg/dL) & $92,75 \pm 9,69$ & $112,25 \pm 10,79$ & 0,200 \\
Triglicerídeos (mg/dL) & $89,00 \pm 5,69$ & $101,87 \pm 7,03$ & 0,177 \\
Colesterol total (mg/dL) & $140,75 \pm 22,30$ & $213,37 \pm 18,29$ & 0,025 \\
HDL (mg/dL) & $41,75 \pm 6,54$ & $75,50 \pm 7,69$ & 0,005 \\
VLDL (mg/dL) & $17,62 \pm 1,18$ & $20,25 \pm 1,36$ & 0,167 \\
LDL (mg/dL) & $81,38 \pm 15,67$ & $118,12 \pm 18,90$ & 0,157 \\
\hline
\end{tabular}

Valores representados em média \pm erro padrão da média.

Teste $t$ de Student.
( $p=0,814)$, com densidade de volume de $63,75 \%$ no grupo que recebeu dieta hiperlipídica e 59,25\% no controle.

No TOTG, assim como no teste de glicemia de jejum, não houve diferença significante na comparação entre os grupos controle e experimental (Figuras 2A, 2B e 2C), cabendo destacar que, nos tempos 15 e 30 minutos, houve um pico de glicemia maior no grupo controle do que no experimental, embora sem diferença estatística.

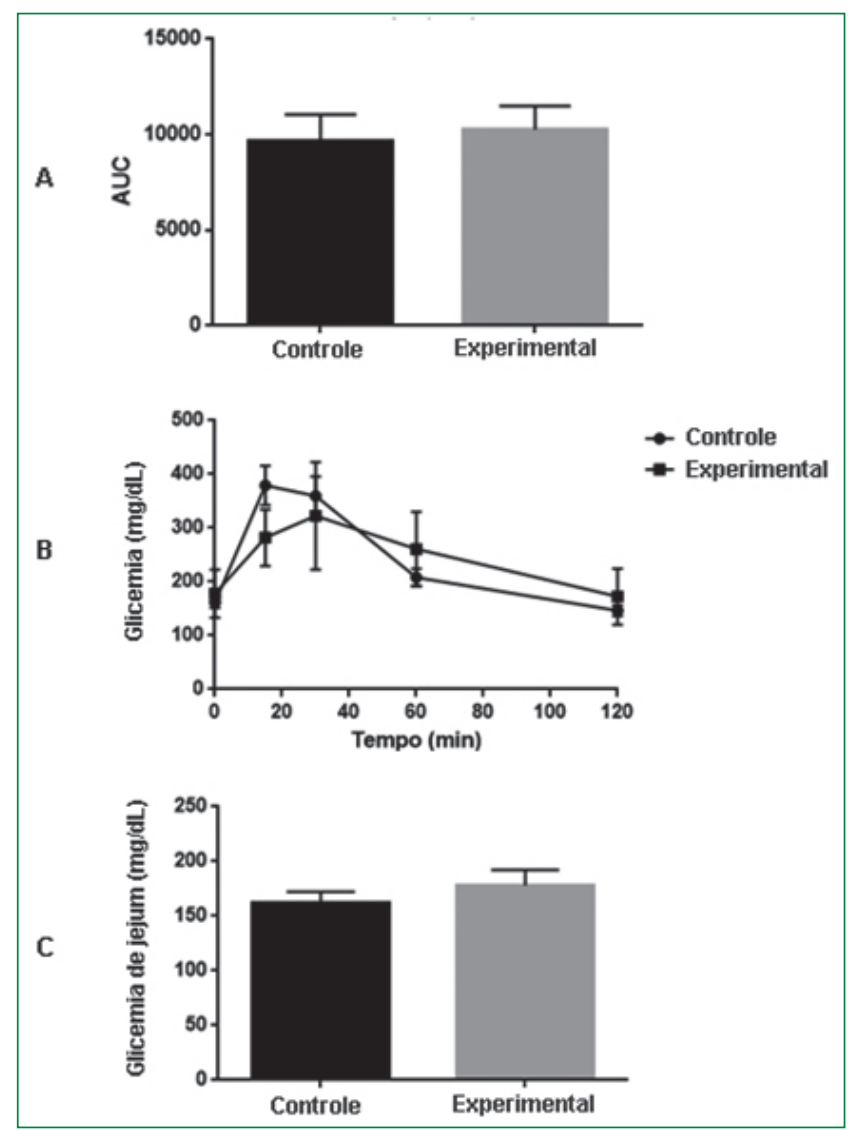

Figura 2. Área sobre a curva $(\mathrm{AUC})$ obtida no teste de tolerância à glicose (TOTG) (A), curva glicêmica (B) e glicemia de jejum (C). Valores representados em média \pm erro padrão da média.

\section{DISCUSSÃO}

Os resultados do presente estudo apontam que a modificação de uma dieta comercial específica para roedores, a partir do acréscimo de banha, apresentou baixo custo e boa aceitação pelos roedores, sendo capaz de alterar o peso corporal em tempo relativamente curto, porém alterou apenas parcialmente outros parâmetros avaliados neste estudo, especialmente no que se refere à alteração da glicemia e gordura hepática.

Sabe-se que o balanço energético sofre forte influência do estilo de vida, seja pelo padrão de consumo alimentar ou 
pelas atividades motoras cotidianas. Ele corresponde à razão entre a quantidade de energia metabolizável ingerida e a quantidade gasta, podendo ser neutro, negativo ou positivo. A obesidade tem base etiológica no balanço energético positivo, que promove ganho excessivo de massa adiposa corporal ${ }^{19}$.

As linhagens isogênicas são obtidas a partir do cruzamento de animais consanguíneos por 20 gerações consecutivas, permitindo a criação de populações estáveis e geneticamente homogêneas. Devido à uniformidade genética, a utilização de camundongos da linhagem isogênica C57BL/6 torna mais propício o desenvolvimento da obesidade. Além disso, a ausência de variáveis genéticas entre os animais torna possível a utilização de um menor o número amostral para um experimento, coexistindo apenas as variáveis ambientais ou experimentais ${ }^{20}$, o que diminui os custos com o experimento.

Os lipídeos representam a maior fonte energética, contendo 9 kcal por grama em comparação a 4 kcal por grama de carboidratos e proteínas ${ }^{21}$, por isso dietas hiperlipídicas são frequentemente utilizadas na tentativa de mimetizar a obesidade em modelos animais, de maneira semelhante à doença humana ${ }^{22}$. A densidade energética da dieta hiperlipídica é maior que da dieta padrão ${ }^{23}$, o que explica o fato do consumo de ração do grupo experimental ter sido menor que o do grupo controle, porém com maior ganho de peso nesse grupo, resultando em diferença significante no CEA.

No presente trabalho, a utilização da dieta hiperlipídica induziu a obesidade, com aumento da adiposidade visceral, verificada pela diferença significativa no peso dos sítios de tecido adiposo epididimal, mesentérico, retroperitoneal e perirrenal, do somatório dos sítios de tecido adiposo, no Índice de Adiposidade e, a partir da quinta semana de consumo da dieta hiperlipídica, do peso dos animais. O aumento da adiposidade visceral está associado à diminuição do HDL, hipertrigliceridemia, aumento da Apo B e das LDL pequenas e densas, e níveis de LDL normais ou marginalmente anormais, sendo que este tipo de dislipidemia aumenta consideravelmente o risco de doenças cardiovasculares ${ }^{24}$.

Experimento com camundongos C57BL6/J verificou que uma dieta hiperlipídica, composta por $42 \%$ de lipídeos, promoveu aumento significante na massa corporal desse grupo em comparação ao grupo padrão ${ }^{11}$. Em outro experimento, camundongos C57BL/6 alimentados com dieta hiperlipídica apresentaram aumento significativo de peso corpóreo, quando comparados aos controles, a partir de 8 meses de indução ${ }^{25}$. Em camundongos Swiss a dieta hiperlipídica também promoveu aumento significante de massa corpórea, bem como da massa de tecido adiposo epididimal, retroperitoneal e perirrenal, em relação ao grupo controle ${ }^{23}$.

Além disso, a análise histológica do tecido adiposo mostrou um aumento significante da área dos adipócitos no grupo de dieta hiperlipídica, assim como verificado por Ravagnani et al. ${ }^{26}$, em estudo com ratos alimentados com dieta hiperlipídica contendo chocolate. Na obesidade ocorre uma hipertrofia dos adipócitos, sendo que esse processo acarreta um aumento da infiltração de macrófagos e da produção das citocinas pró-inflamatórias, como o fator de necrose tumoral (TNF- $\alpha$ ) e interleucina 6 (IL-6). Ocorre também elevação na liberação de ácidos graxos livres e desregulação na secreção de adiponectina, resistina e leptina. A secreção alterada destas adipocinas pode elevar a ingestão alimentar e reduzir o gasto energético ${ }^{1}$. Estas adipocinas também podem exacerbar o processo inflamatório no organismo e estão envolvidas no desenvolvimento da resistência à insulina ${ }^{27}$.

Com o maior fluxo de ácidos graxos livres há a perpetuação da resistência à insulina ${ }^{28}$ e estes são utilizados na produção hepática de VLDL, lipoproteína rica em triglicerídeos, favorecendo o aparecimento da hipertrigliceridemia. Além disso, essa lipoproteína será metabolizada em LDL, que favorece o processo de aterogênese ${ }^{29}$. Nesse estudo, o aumento do tecido adiposo, e consequentemente da liberação de ácidos graxos livres, nos animais do grupo que recebeu dieta hiperlipídica causou a elevação dos níveis de LDL, VLDL e triglicerídeos, porém sem diferença significativa em relação ao grupo controle.

Houve também uma elevação significante do colesterol total e do HDL em relação ao controle, em conformidade com os achados de Almeida et al. ${ }^{30}$, e o Índice Aterogênico não apresentou diferença significante. Os ácidos graxos saturados, presentes em grande quantidade na ração hiperlipídica oferecida ao grupo experimental, são um dos componentes dietéticos que mais aumentam o LDL, promovendo também aumento do $\mathrm{HDL}$, porém sem alterar o índice aterogênico ${ }^{31}$, mostrando que o aumento do HDL no grupo experimental não diminuiu o risco cardiovascular nesse grupo.

Os grupos experimental e controle apresentaram esteatose hepática, sendo classificada em moderada (de 33 a 66\%) em $a_{b} b^{32}$ e sem diferença significante entre eles. A esteatose hepática é uma condição onde há acúmulo de gordura no fígado, intimamente relacionada com a obesidade central, resistência à insulina, hipertrigliceridemia e hiperglicemia de jejum. Ela ocorre quando a síntese de ácidos graxos pelos hepatócitos excede o catabolismo. Assim, as possíveis causas de acúmulo de lipídeos no fígado são: (a) aumento da distribuição e absorção de ácidos graxos de cadeia longa (AGCL) para os hepatócitos devido ao excesso de ingestão 
ou liberação a partir de tecido adiposo; (b) aumento da síntese hepática de AGCL e triglicerídeos; (c) falha do VLDL na síntese e exportação de triglicerídeos; e/ou (d) falha na eliminação dos AGCL devido à $\beta$-oxidação mitocondrial hepática ${ }^{33}$. Um tempo maior de estudo e aumento do estresse oxidativo, considerado o principal gatilho na evolução da esteatose hepática para esteato-hepatite não alcoólica e cirrose $^{34}$, talvez promovesse diferença significante entre os grupos, visto que houve aumento significativo dos níveis de colesterol total e aumento, sem diferença estatística, dos níveis de LDL, VLDL e triglicerídeos, no grupo experimental.

A evolução do diabetes mellitus passa por estágios intermediários, como a glicemia de jejum alterada e a intolerância à glicose e esses estágios são decorrentes da resistência à insulina combinada com a disfunção da célula $\beta$ pancreática ${ }^{35}$. Na resistência à insulina, as células $\beta$ do pâncreas aumentam a produção de insulina como mecanismo compensatório, tentando, assim, normalizar os níveis de glicose no sangue, até que se verifica uma redução permanente da sensibilidade à insulina, com concomitante elevação da glicemia, o que leva ao desenvolvimento do diabetes mellitus ${ }^{36}$.

Atualmente, para o diagnóstico do diabetes mellitus podem ser utilizados os sintomas de poliúria, polidipsia e perda ponderal, o teste de glicemia de jejum e o TOTG ${ }^{35}$. Apesar de não ter apresentado diferença significante na área da curva do TOTG e no teste de glicemia de jejum, o estudo verificou uma tendência ao desenvolvimento de diabetes mellitus nos camundongos $\mathrm{C} 57 \mathrm{BL} / 6$, visto que a hiperglicemia de jejum fornece evidência tanto de resistência à insulina quanto de disfunção de célula beta ${ }^{37}$, entretanto ressalta-se a necessidade de outras intervenções no modelo experimental para se alcançar tais objetivos. As dietas hipercalóricas, como demonstrado em artigo de revisão, são mais propensas a promover aumento significante da glicose plasmática em roedores ${ }^{8}$.

O modelo de estudo aqui apresentado poderia trazer maiores informações para a literatura científica se outros grupos de estudo tivessem sido formados, especialmente utilizando a ração AIN-937, visto que a grande maioria dos estudos publicados utiliza essa ração modificada com o acréscimo de gordura para produzir alterações metabólicas em roedores, mas nem sempre apresentam resultados eficazes em tal indução, pois a própria ração AIN padrão gera alterações metabólicas ${ }^{38}$. Destaca-se que a ração AIN93 apresenta alto custo se comparada a ração comercial utilizada neste estudo. Além disso, como os animais foram mantidos em colônias, não foi possível determinar a quantidade individualizada de ração consumida por cada animal.
Conclui-se que este modelo induziu a obesidade em um curto período de tempo e promoveu aumento da adiposidade visceral, com dieta de baixo custo. Entretanto, são necessários outros estudos visando melhorar sua eficiência e, assim, conseguir alterar de forma completa os lipídeos sanguíneos e a glicemia nos camundongos C57BL/6.

\section{REFERÊNCIAS}

1. Galic S, Oakhill JS, Steinberg GR. Adipose tissue as an endocrine organ. Mol Cell Endocrinol. 2010;316(2):129-39. https://doi. org/10.1016/j.mce.2009.08.018

2. Lee S, Bacha F, Silva AA. Waist circumference, blood pressure, and lipid components of the metabolic syndrome. J Pediatr. 2006;149(6):809-16. https://doi.org/10.1016/j.jpeds.2006.08.075

3. Sant'Anna S, Caiado NM, Silva LJ. Neuromodulação hipotalâmica: uma proposta terapêutica para obesidade. Rev Med Saude Brasília. 2014;3(2):192-204.

4. Ministério da Saúde (BR), Secretaria de Vigilância em Saúde, Departamento de Vigilância de Doenças e Agravos não Transmissíveis e Promoção da Saúde. Vigitel Brasil 2015: estimativas sobre frequência e distribuição sociodemográfica de fatores de risco e proteção para doenças crônicas nas capitais dos 26 estados brasileiros e no Distrito Federal em 2015. Brasília: Ministério da Saúde; 2016.

5. Soares FLP. Efeitos e mecanismos de ação da exclusão dietética de glúten de trigo na obesidade e resistência à insulina experimentais [tese]. Minas Gerais: Universidade Federal de Minas Gerais, Instituto de Ciências Biológicas; 2012.

6. Mosca PRF, Silveira PP, Werlang ICR, Goldani MZ. Obesidade e genética. Rev HCPA. 2012;32(3):318-31.

7. Reeves PG, Nielsen FH, Fahey GC JR. AIN-93 purified diets for laboratory rodents: final report of the American Institute of Nutrition ad hoc writing committee on the reformulation of the AIN-76A rodent diet. J Nutr. 1993;123(11):1939-51. https://doi.org/10.1093/ jn/123.11.1939

8. Rosini TC, Silva ASR, Moraes C. Obesidade induzida por consumo de dieta: modelo em roedores para o estudo de distúrbios relacionados com a obesidade. Rev Assoc Med Bras. 2012;58(3):383-7. https:// doi.org/10.1590/S0104-42302012000300021

9. Soares FLP, Santana L, Pinto LA, Dutra TS, Freitas KC, Kassuya CAL. Novo modelo experimental de síndrome metabólica induzida por dieta. In: 12을 Congresso da Sociedade Brasileira de Alimentação e Nutrição; 2013; Foz do Iguaçu. São Paulo: Nutrire - Sociedade Brasileira de Alimentação e Nutrição - SBAN; 2013. p. 234-4.

10. Chu HF, Pan MH, Ho CT, Tseng YH, Wang WW, Chau CF. Variations in the Efficacy of Resistant Maltodextrin on Body Fat Reduction in Rats Fed Different High-Fat Models. J Agric Food Chem. 2014;62(1): 192-7. https://doi.org/10.1021/jf404809v

11. Moreira AS. Dietas ricas em gordura, frutose e sacarose: alterações no fígado [tese]. Rio de Janeiro: Universidade Estadual do Rio de Janeiro, Instituto de Biologia Roberto Alcântara Gomes; 2014.

12. Freitas KC, Amancio OMS, Morais MB. High-performance inulin and oligofructose prebiotics increase the intestinal absorption of iron in rats with iron deficiency anaemia during the growth phase. Br J Nutr. 2012;108(6):1008-16. https://doi.org/10.1017/ S0007114511006301 
13. Machado H, Pinheiro HS, Terra MM, Guerra MO, de Paula RB, Peters VM. Dissociação da resposta anti-hipertensiva e metabólica à losartana e espironolactona em ratos com síndrome metabólica experimental. J Bras Nefrol. 2012;34(4):328-36. https://doi. org/10.5935/0101-2800.20120021

14. Taylor BA, Phillips SJ. Detection of obesity QTLs on mouse chromosomes 1 and 7 by selective DNA pooling. Genomics. 1996;34(302):389-98. https://doi.org/10.1006/geno.1996.0302

15. Mendonça FS, Evêncio-Neto J, Simões MJ, Simões RS, Baratella Evêncio L. Freqüência de hipoplasia ovariana em vacas abatidas em Rondônia. Arq Inst Biol. 2006;68(Supl):267-70.

16. Aguila MB, Pinheiro AR, Parente LB, Mandarim-de-Lacerda CA. Dietary effect of different high-fat diet on rat liver stereology. Liver Int. 2003;23:363-70. https://doi.org/10.1034/j.1478-3231. 2003.00858.x

17. Catta-Preta M, Mendonça LS, Fraulob-Aquino J, Aguila MB, Mandarim-de-Lacerda CA. A critical analysis of three quantitative methods of assesment of hepatic steatosis in liver biopsies. Virchows Arch. 2011;459:477-85. https://doi.org/10.1007/s00428-0111147-1

18. Florence NT, Benoit MZ, Jonas K, Alexandra T, Désiré DDP, Pierre K, Théophile D. Antidiabetic and antioxidant effects of Annona muricata (Annonaceae), aqueous extract on streptozotocin-induced diabetic rats. J Ethnopharmacol. 2014;151(2):784-90. https://doi. org/10.1016/j.jep.2013.09.021

19. Carvalho KMB, Dutra ES. Obesidade. In: Cuppari L. Nutrição clínica no adulto. 3a ed. Barueri, SP: Manole; 2014. p. 185-214.

20. Ferreira LM, Hochman B, Barbosa MVJ. Modelos experimentais em pesquisa. Acta Cir Bras. 2005;20(2):28-34. https://doi.org/10.1590/ S0102-86502005000800008

21. Avesani CM, Santos NSJ, Cuppari L. In: Cuppari L. Nutrição Clínica no Adulto. 3a ed. Barueri, SP: Manole; 2014. p. 45-62.

22. Buettner R, Schölmerich J, Bollheimer LC. High-fat diets: modeling the metabolic disorders of human obesity in rodents. Obesity. 2007;15(4):798-808. https://doi.org/10.1038/oby.2007.608

23. White PAS, Cercato LM, Araújo JMD, Souza LA, Soares AF, Barbosa APO, R Neto JM, Marçal AC, Machado UF, Camargo EA, Santos MRV, Brito LC. Modelo de obesidade induzida por dieta hiperlipídica e associada à resistência à ação da insulina e intolerância à glicose. Arq Bras Endocrinol Metab. 2013;57(5):339-45. https://doi. org/10.1590/S0004-27302013000500002

24. Barroso SG, de Abreu VG, Francischetti EA. A Participação do tecido adiposo visceral na gênese da hipertensão e doença cardiovascular aterogênica. Arq Bras Cardiol. 2002;78(6):618-30. https://doi. org/10.1590/S0066-782X2002000600012

25. Kiperstok AC. Avaliação longitudinal da função cardíaca em modelo experimental de diabetes tipo 2 induzido por dieta hipergordurosa em camundongo [dissertação]. Salvador: Fundação Oswaldo Cruz; 2010.

26. Ravagnani FCP, Ravagnani CFC, Braga Neto JA, Voltarelli FA, Zavala AAZ, Habitante CA, Inouye CM. Efeito de dietas hiperlipídicas com extrato de baru e chocolate sobre a área de adipócitos de ratos submetidos ao exercício físico. Rev Bras Med Esporte. 2012;18(3):190-4. https://doi.org/10.1590/S151786922012000300011

27. Gutierrez DA, Puglisi MJ, Hasty AH. Impact of increased adipose tissue mass on inflammation, insulin resistance, and dyslipidemia. Curr Diab Rep. 2009;9(1):26-32. https://doi.org/10.1007/s11892009-0006-9

28. Martínez G, Alonso R, Novik V. Síndrome metabólico: bases clínicas y fisiopatológicas para un enfoque terapéutico racional. Rev Méd Chile. 2009;137(5):685-94. https://doi.org/10.4067/S003498872009000500014

29. Fonseca FAH. Síndrome metabólica sem obesidade central: mito ou realidade? Rev Bras Hipertens. 2008;15(3):168-9.

30. Almeida MEF, Medeiros RS, Figueiredo FJB, Coelho EJB, Sena MPT. Efeitos do estresse auditivo e da dieta hipercalórica sobre o peso corporal, lipídios e glicemia de ratos wistar. Alim Nutr. 2011;22(3):359-65.

31. Santos RD, Gagliardi ACM, Xavier HT, Magnoni CD, Cassani R, Lottenberg AMP,Casella Filho A, Araújo DB, Cesena FY, Alves RJ, Fenelon G, Nishioka SAD, Faludi AA, Geloneze B, Scherr C, Kovacs C, Tomazzela C, Carla C, Barrera-Arellano D, Cintra D, Quintão E, Nakandakare ER, Fonseca FAH, Pimentel I, Santos JE, Bertolami MC, Rogero M, Izar MC, Nakasato M, Damasceno NRT, Maranhão R, Cassani RSL, Perim R, Ramos S; Sociedade Brasileira de Cardiologia. I Diretriz sobre o consumo de gorduras e saúde cardiovascular. Arq Bras Cardiol. 2013;100(1 Supl. 3):1-40. https://doi.org/10.5935/ abc.2013S003

32. Almeida e Borges VF, Diniz ALD, Cotrim HP, Rocha HLOG, Salomão FC. Dopplerfluxometria da veia hepática em pacientes com esteatose não alcoólica. Radiol Bras. 2011;44(1):1-6. https://doi. org/10.1590/S0100-39842011000100005

33. Takahashi Y, Soejima Y, Fukusato T. Animal models of nonalcoholic fatty liver disease/nonalcoholic steatohepatitis. World J Gastroenterol. 2012;18(19):2300-8. https://doi.org/10.3748/wjg.v18.i19.2300

34. Carvalheira JBC, Saad MJA. Doenças associadas à resistência à insulina/ hiperinsulinemia, não incluídas na síndrome metabólica. Arq Bras Endocrinol Metab. 2006;50(2):360-7. https://doi. org/10.1590/S0004-27302006000200022

35. Sociedade Brasileira de Diabetes. Diretrizes da Sociedade Brasileira de Diabetes: 2013-2014. São Paulo: AC Farmacêutica; 2014.

36. Chiarelli F, Marcovecchio ML. Insulin resistance and obesity in childhood. Eur J Endocrinol. 2008;159(1):67-74. https://doi. org/10.1530/EJE-08-0245

37. Davies MJ, Raymond NT, Day JL, Hales CN, Burden AC. Impaired glucose tolerance and fasting hyperglycaemia have different characteristics. Diabet Med. 2000;17(6):433-40. https://doi. org/10.1046/j.1464-5491.2000.00246.x

38. Ferreira PA, Salame JP, Bastos RF, Francisco LS, Felix AOC, Felix SR, Nobre MO. Considerações sobre a dieta ain-93m elaborada para modelos biológicos. Resbcal. 2015;3(2):103-8. 